\title{
Making urinary extracellular vesicles a clinically tractable source of biomarkers for inherited tubulopathies using a small volume precipitation method: proof of concept
}

\author{
Timothy Lee Williams $^{1}$ [ $\cdot$ Carlos Bastos $^{1} \cdot$ Nuno Faria $^{1} \cdot$ Fiona Eve Karet Frank $\left.\right|^{2}$
}

Received: 10 May 2019 / Accepted: 24 September 2019 / Published online: 4 October 2019

(C) The Author(s) 2019

\begin{abstract}
Biomarkers of inherited tubulopathies would be useful for clarifying diagnoses in patients where genetic screening is not readily available or where disease-attributable mutations are not found. Urinary extracellular vesicles (uEVs) obtained by ultracentrifugation can be used as a source of biomarkers for inherited tubulopathies such as Gitelman Syndrome (GS), however, ultracentrifugation requires costly equipment and is thus not usually accessible. In contrast, precipitation methods can extract uEVs using standard laboratory centrifuges, thus making uEVs extracted by this method clinically tractable as a source of biomarkers for GS and other inherited tubulopathies. Here we optimise a precipitation method for extracting urinary extracellular vesicles (uEVs) and provide proof of concept that these uEVs are a source of biomarkers using GS an exemplar tubulopathy. For method optimisation, uEVs were precipitated from fresh and frozen (for up to 6 years), small volume (1-2 mL) urine samples from healthy volunteers and GS patients. Nanoparticle tracking analysis was used to calculate the concentration of uEVs. Thiazide sensitive sodium-chloride cotransporter (NCC) content was determined by densitometry of Western blots. NCC content of uEVs was lower in GS patients $(\mathrm{n}=11)$ than healthy volunteers $(\mathrm{n}=12 ; \mathrm{P}=0.001)$. Three of four patients clinically suspected for GS, in whom only a single SLC12A3 mutation was identified, had lower uEV NCC content than all healthy volunteers tested. In the clinical setting, sufficient uEVs can be extracted from frozen, small volume urine samples using precipitation methods to distinguish patients with GS from healthy volunteers, and thus this source of uEVs could be utilised as an additional diagnostic test for GS and similar disorders.
\end{abstract}

Keywords Gitelman Syndrome · Precipitation · Exosome

\section{Introduction}

Inherited tubulopathies are rare transporter disorders of renal tubular epithelia causing defective renal handling of electrolytes, amino-acids, water and/or glucose. In Gitelman Syndrome (GS; loss of thiazide-sensitive sodium-chloride

Electronic supplementary material The online version of this article (https://doi.org/10.1007/s40620-019-00653-8) contains supplementary material, which is available to authorized users.

Timothy Lee Williams

timwilliams@cantab.net

1 Department of Veterinary Medicine, University of Cambridge, Madingley Road, Cambridge CB3 OES, UK

2 Department of Medical Genetics and Division of Renal Medicine, University of Cambridge and Cambridge University Hospitals Foundation Trust, Cambridge, UK cotransporter function), genetic testing has a reported clinical sensitivity of only $65-80 \%$ [1], therefore identification of biomarkers of inherited tubulopathies (including GS) would be useful in patients where there is clinical doubt and/or where genetic testing is not readily available.

Urinary extracellular vesicles (uEVs) are a potentially rich source of biomarkers for tubulopathies, since their membranes are composed of apical proteins from all nephron segments [2]. Thiazide-sensitive sodiumchloride cotransporter (NCC) abundance within uEVs of GS patients obtained by ultracentrifugation is reduced on Western blot [3, 4]; however, ultracentrifugation requires costly equipment, and so this method of $\mathrm{uEV}$ extraction would not be easily accessible to clinicians for diagnostic purposes. Commercially available kits allow extraction of uEVs by precipitation using standard laboratory centrifuges; this might make the use of uEVs as a source of biomarkers more widely applicable and clinically tractable. 
Here we provide proof of concept that $u E V s$ extracted by precipitation from small-volume urine samples can be so used, using GS as an exemplar tubulopathy. Therefore, this technique could be adapted to yield a clinically tractable additional diagnostic test for GS and potentially other tubulopathies.

\section{Materials and methods}

The Exiqon miRCURY Exosome Isolation Kit (\#300102) was used according to manufacturer protocol for extraction of uEVs, with the addition of a filtration step $(0.22 \mu \mathrm{m}$ filter, Millipore Stericup 250) after harvesting of the initial supernatant, and resuspension of the final pellet containing uEVs in $50 \mu \mathrm{L}$ of resuspension buffer (rather than $100 \mu \mathrm{L}$ ). We evaluated a modification to the standard protocol with the aim of increasing uEV yield; fresh urine samples without dipstick abnormalities (Siemens Multistix 10SG) were collected from healthy volunteers into sterilised glass bottles and incubated with precipitation buffer, either overnight or for the manufacturer-recommended time of $60 \mathrm{~min}$, at $4{ }^{\circ} \mathrm{C}$. Total protein concentrations of uEV preparations were measured using a bioinchoninic acid (BCA) method (Pierce ${ }^{\mathrm{TM}}$ BCA Protein Assay Kit, Thermo Scientific), and particle numbers determined by nanoparticle tracking analysis on a Nanosight NS500 (Supplementary Figure 1, Malvern, UK). Comparisons between $60 \mathrm{~min}$ and overnight incubation, and precipitation and ultracentrifugation were made using the Wilcoxon signed-rank test; data are presented as median [range].

To provide proof of concept that uEVs could be used as a source of biomarkers in inherited tubulopathies, azide-preserved small volume (1-2 $\mathrm{mL})$ urine samples from healthy volunteers and patients with GS, stored at $-80{ }^{\circ} \mathrm{C}$, were obtained from the sample bank of the Cambridge Renal Genetic and Tubular Disorders service. Samples were collected under Cambridgeshire Research Ethics Committee approval (08-H0306-62) with informed consent of participants. Protease inhibitors (Roche cOmplete, EDTA-free) were added at the point of thawing. Since cooling increases uromodulin polymerisation [5], samples were alkalinised by addition of $1 \mathrm{M} \mathrm{NaOH}$ to $\mathrm{pH} 8.1$ (determined using MColorpHast $^{\mathrm{TM}} \mathrm{pH}$ indicator strips) prior to processing, to reverse this tendency [6] and maximise uEV yield.

To exclude an adverse effect of alkalinisation on protein and uEVs extraction by precipitation, further fresh urine samples were collected from healthy volunteers $(n=9)$ and divided into two aliquots, one of which was alkalinised (as per protocol above) prior to processing. Protein and particle yields were compared using the Wilcoxon signed rank test.

\section{Results}

Overnight incubation of samples with precipitation buffer significantly increased particle concentration within $\mathrm{UEV}$ preparations $\left(1.6 \times 10^{10} / \mathrm{mL}\left[0.3-3.5 \times 10^{10} / \mathrm{mL}\right]\right.$ vs. $0.07 \times 10^{10} / \mathrm{mL}$ $\left.\left[0.03-0.2 \times 10^{10} / \mathrm{mL}\right] ; \mathrm{P}=0.03\right)$, hence overnight incubation was performed thereafter. Total protein content of $\mathrm{uEV}$ preparations was unchanged (3.8 [3.1-5.1] vs. $3.3[1.8-6.4] \mu \mathrm{g} /$ $\mathrm{mL} ; \mathrm{n}=6, \mathrm{P}=0.3)$. The presence of $\mathrm{uEVs}$ within preparations obtained by precipitation was confirmed by Western blotting for CD9 and TSG101 (Supplementary Figure 2).

The precipitation method yielded a similar quantity of protein and uEVs (on a per $\mathrm{mL}$ urine basis) compared to ultracentrifugation (as per our previously published protocol [7]); protein contents were $0.7-10.8 \mu \mathrm{g}(0.7-10.8 \mu \mathrm{g} / \mathrm{mL}$ urine) and $12.1-50.8 \mu \mathrm{g}(0.2-0.8 \mu \mathrm{g} / \mathrm{mL}$ urine $)$ in uEV preparations produced by precipitation and ultracentrifugation respectively $(\mathrm{n}=3 ; \mathrm{P}=0.2)$. Total number of $\mathrm{uEVs}$ from these preparations were $6.2-28.0$ billion and 1.0-27.8 billion particles $/ \mathrm{mL}$ urine respectively $(n=3 ; P=0.4)$.

Protein content of $\mathrm{uEV}$ preparations obtained from frozen samples (used as surrogate marker of $\mathrm{uEV}$ quantity) ranged from 1.2 to $30.9 \mu \mathrm{g}$. Thirty microlitre aliquots of uEV preparations were reduced and separated by $4-12 \%$ SDS-PAGE (NuPAGE, Thermo Fisher) prior to transfer to nitrocellulose membranes (Bio-Rad). Membranes were blocked with 5\% skimmed milk in $0.1 \%$ PBS-Tween 20, and incubated with primary (anti-NCC [Abcam, ab95032], anti-CD9 [Abcam, ab92729] or anti-TSG101 [Abcam, ab83]) and secondary (IRDye ${ }^{\circledR}$ or HRP-conjungated) antibodies (Dako). Bands of appropriate size were observed using an infrared imaging system (LICOR Odyssey) or on radiographic film (following addition of chromographic reagent), and densitometry performed using ImageJ software (NIH, USA). Comparisons between groups were made using the Mann-Whitney U test.

NCC content of uEVs, normalised to quantity of protein loaded, was significantly lower in GS patients $(n=11)$ than healthy volunteers $(n=12 ; P=0.001$, Fig. 1$)$. Three of four patients clinically suspected to have GS, but in whom only a single SLC12A3 mutation was identified, also had lower uEV NCC content than all healthy volunteers tested (Fig. 1).

Alkalinisation did not adversely affect either protein concentration (non-alkalinised $3.9 \mu \mathrm{g} / \mathrm{mL}$ [1.0-6.6 $\mu \mathrm{g} / \mathrm{mL}$ ], alkalinised $4.8 \mu \mathrm{g} / \mathrm{mL}$ [0.9-9.1 $\mu \mathrm{g} / \mathrm{mL}] ; \mathrm{n}=9 ; \mathrm{P}=0.25$ ) or particle yield (non-alkalinised $2.3 \times 10^{10}\left[2.4 \times 10^{9}-4.2 \times 10^{10}\right]$ particles/mL, alkalinised $1.4 \times 10^{10}\left[5.3 \times 10^{8}-3.2 \times 10^{10}\right]$ particles/mL; $\mathrm{P}=0.13$ ). 

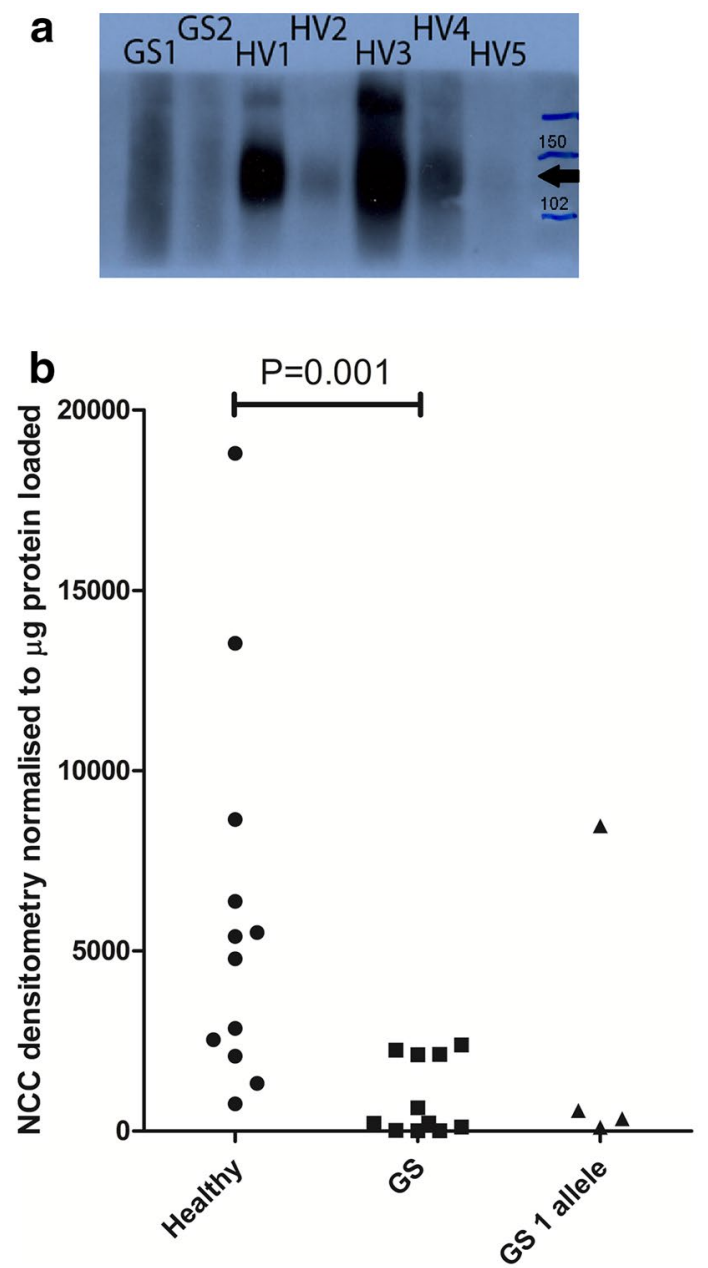

Fig. 1 a Representative western blot of urinary extracellular vesicle $(\mathrm{uEV})$ preparations, obtained from small volume urine samples from healthy volunteers (HV) and patients with genetically confirmed Gitelman Syndrome (GS), for the thiazide sensitive sodiumchloride cotransporter (NCC). Expected size is $\sim 111 \mathrm{kDa}$ (indicated by the black arrow). Molecular size markers (in $\mathrm{kDa}$ ) are shown in the right hand lane. uEVs were extracted from 1 to $2 \mathrm{~mL}$ urine samples, previously stored at $-80{ }^{\circ} \mathrm{C}$, by precipitation. b Scatter graph showing densitometry results from Western blot for the thiazide sensitive sodium-chloride cotransporter (NCC) in urinary extracellular vesicles (uEVs) obtained from small volume urine samples from healthy volunteers, patients with confirmed Gitelman Syndrome (GS) based on genetic testing, and patients clinically suspected to have GS for which only one known mutation of SLC12A3 could be identified (GS 1 allele). Densitometry readings for NCC were normalised to the amount of protein loaded (in $\mu \mathrm{g}$ ). $\mathrm{uEV}$ NCC content was significantly lower in GS patients compared to healthy volunteers $(P=0.001)$

\section{Discussion}

This study demonstrates that sufficient uEVs can be extracted from small volume urine samples by precipitation methods for immunoblotting studies. To the authors' knowledge, this is the first study to demonstrate that the quantity of NCC present within uEVs extracted by precipitation is lower in patients with GS than in healthy volunteers. Furthermore, the abundance of NCC within uEVs of most patients suspected of GS but without a confirmed genetic diagnosis was also low, thus indicating that this method could be a useful additional test for GS in patients in which a diagnosis is not reached by genetic testing. These findings concur with those of a previous study which evaluated uEVs extracted from GS patients by ultracentrifugation [3]. Further studies to compare the uEVs extracted using both techniques would be of potential interest, but clinicians are unlikely to have routine access to ultracentrifuges, so that technique is not generalizable. The main advantage of the precipitation technique validated here is that extraction of uEVs can be performed using standard laboratory centrifuges, thus making uEVs a widely accessible and clinically tractable source of biomarkers for GS and other inherited tubulopathies. These are encouraging data, and further refinement and investigation of this technique as a diagnostic test for GS is warranted.

Normalised NCC abundance within uEV preparations of some GS patients was similar to healthy volunteers, perhaps reflecting expression of some mutant NCC proteins at the apical membrane, and thus within uEVs [8]. It is recognised that suitable normalisation of uEVs is problematic because no single parameter can specifically quantify EVs; and EV subtypes from different nephron segments will express different markers [9]. We used total protein for this purpose because it is a clinically accessible surrogate marker for all $\mathrm{uEVs}$, and our aim was to demonstrate proof of concept for a clinically tractable test. Although we acknowledge that total protein is a non-specific surrogate marker of uEV number, we would have expected that using it would if anything decrease the likelihood of identifying a significant difference between the normalised NCC content of healthy volunteers and GS patients as in the present study. The main limitation of our study was the relatively small number of GS patient samples included in the proof of concept study, which reflects the ultra-rarity of GS in the population (predicted prevalence approximately 1:40,000 [1]). However, our study was still adequately powered to demonstrate a significant difference in the NCC content of uEVs between GS patients and healthy volunteers.

Our technique could also improve characterisation of the molecular bases of other inherited renal tubulopathies. For example, excretion of aquaporin-2 (AQP2) within uEVs correlates with AQP2 expression in the kidneys of humans and rats $[4,10]$, suggesting that the $\mathrm{uEV}$ proteome is a surrogate marker for expression of apical proteins within the nephron. That said, hydration status affects AQP2 expression, so even this protein would not be reliable as a denominator of $\mathrm{EV}$ amount in healthy volunteers. Excretion of various proteins within uEVs of humans and rats has been reported [4, 10], and the precipitation techniques utilised here will allow future studies of the $\mathrm{uEV}$ proteome to be performed using 
urine samples of lower volume. This could be also particularly beneficial when studying animal models in which urine volumes are low.

\section{Compliance with ethical standards}

Conflict of interest On behalf of all authors, the corresponding author states that there is no conflict of interest.

Ethical approval All procedures performed in studies involving human participants were in accordance with the ethical standards of the institutional and/or national research committee (Cambridgeshire Research Ethics Committee approval 08-H0306-62) and with the 1964 Helsinki Declaration and its later amendments or comparable ethical standards.

Informed consent Informed consent was obtained from all individual participants included in the study.

Open Access This article is distributed under the terms of the Creative Commons Attribution 4.0 International License (http://creativeco mmons.org/licenses/by/4.0/), which permits unrestricted use, distribution, and reproduction in any medium, provided you give appropriate credit to the original author(s) and the source, provide a link to the Creative Commons license, and indicate if changes were made.

\section{References}

1. Blanchard A et al (2017) Gitelman syndrome: consensus and guidance from a Kidney Disease: Improving Global Outcomes (KDIGO) Controversies Conference. Kidney Int 91(1):24-33
2. De Palma G, Sallustio F, Schena FP (2016) Clinical application of human urinary extracellular vesicles in kidney and urologic diseases. Int J Mol Sci 17(7):1043

3. Corbetta $\mathrm{S}$ et al (2015) Urinary exosomes in the diagnosis of Gitelman and Bartter syndromes. Nephrol Dial Transplant 30(4):621-630

4. Joo KW et al (2007) Reduced urinary excretion of thiazide-sensitive $\mathrm{Na}-\mathrm{Cl}$ cotransporter in Gitelman syndrome: preliminary data. Am J Kidney Dis 50(5):765-773

5. Fernandez-Llama P et al (2010) Tamm-Horsfall protein and urinary exosome isolation. Kidney Int 77(8):736-742

6. Kobayashi K, Fukuoka S (2001) Conditions for solubilization of Tamm-Horsfall protein/uromodulin in human urine and establishment of a sensitive and accurate enzyme-linked immunosorbent assay (ELISA) method. Arch Biochem Biophys 388(1):113-120

7. Gracia T et al (2017) Urinary exosomes contain MicroRNAs capable of paracrine modulation of tubular transporters in kidney. Sci Rep 7:40601

8. De Jong JC et al (2002) Functional expression of mutations in the human $\mathrm{NaCl}$ cotransporter: evidence for impaired routing mechanisms in Gitelman's syndrome. J Am Soc Nephrol 13(6): 1442-1448

9. Thery $\mathrm{C}$ et al (2018) Minimal information for studies of extracellular vesicles 2018 (MISEV2018): a position statement of the International Society for Extracellular Vesicles and update of the MISEV2014 guidelines. J Extracell Vesicles 7(1):1535750

10. Wen $\mathrm{H}$ et al (1999) Urinary excretion of aquaporin-2 in rat is mediated by a vasopressin-dependent apical pathway. J Am Soc Nephrol 10(7):1416-1429

Publisher's Note Springer Nature remains neutral with regard to jurisdictional claims in published maps and institutional affiliations. 